\title{
Comparaison du comportement maternel en bergerie à la parturition chez des brebis primipares ou multipares de race Romanov, Préalpes du Sud et Ile-de-France
}

\author{
P. POINDRON, Ildiko RAKSANYI, P. ORGEUR et P. LE NEINDRE * \\ I.N.R.A., Laboratoire de Comportement animal, Centre de Recherches de Tours, \\ Nouzilly, F 37380 Monnaie \\ *I.N.R.A., Laboratoire de Production de Viande, \\ C.R.Z.V. de Theix, F 63110 Beaumont
}

\section{Résumé}

Le comportement maternel au cours des 3 premières heures de la vie de l'agneau a été comparé chez des brebis lle-de-France (IF), Préalpes du Sud (PA) et Romanov (R). L'étude a porté à la fois sur des femelles primipares et multipares dont la reproduction était synchronisée par l'utilisation d'éponges vaginales imprégnées de FGA suivies d'une injection de PMSG, et dont la parturition était induite par une injection de dexaméthasone (IF : $16 \mathrm{mg}$ à $\mathrm{J} 144$ de gestation ; PA : $12 \mathrm{mg}$ à $\mathrm{J} 143 ; \mathrm{R}: 16 \mathrm{mg}$ à J 140). Chez les brebis multipares, aucune différence très marquée n'a été observée entre les 3 races, à l'exception du léchage de l'agneau, qui a débuté plus tardivement chez les IF que chez les $R$ et les $P A(P<0,05$ dans les 2 cas). Par contre, les différences entre races ont été beaucoup plus marquées chez les femelles agnelant pour la première fois. Les troubles du léchage ou de l'acceptation à la mamelle ont été moins fréquents chez les R (10 p. 100) que chez les IF (50 p. 100) ou les PA (35 p. 100). Le comportement agressif a été plus fréquent chez les PA (29 p. 100 des mères) que chez les IF $(7 \mathrm{p} .100, P<0,05)$ ou les $R$ $(19$ p. 100 , ns). 60 p. 100 des mères $R$ n'ont manifesté aucun trouble de comportement dès la parturition et 3 heures plus tard, 90 p. 100 avaient adopté leur agneau. Chez les brebis IF, ces proportions étaient respectivement de 35 et 61 p. 100 ( $\mathrm{P}<0,05$ dans les 2 cas). Les performances des brebis PA étaient intermédiaires (34 p. 100 et 84 p. 100 respectivement). Donc, d'une façon générale, les brebis primipares manifestent plus fréquemment des troubles de comportement maternel que les brebis multipares. Par ailleurs, les brebis Romanov ont eu un comportement maternel mieux adapté que les 2 autres races, et les Ile-de-France sont apparues comme étant les moins bonnes mères. Il faut souligner aussi que dans les 3 races, de grandes variations individuelles ont été notées : certains animaux ont très bien accepté leur agneau alors que d'autres l'on refusé jusqu'à la fin de l'étude.

Mots clés : Comportement maternel, ovins, expérience, différences raciales.

\section{Summary}

Comparison of maternal behaviour at parturition in primiparous or multiparous ewes of Ile-de-France, Préalpes du Sud and Romanov breeds

We have compared maternal behaviour during the 3 first hours after parturition in Ile-de-France (IF), Préalpes (PA) and Romanov (R) ewes. Both primiparous and multiparous females were studied. Reproduction was fully synchronized (vaginal sponges and PMSG at oestrus). Parturition 
was induced with an injection of dexamethasone (IF : $16 \mathrm{mg}$ on day 144 of gestation; PA : $12 \mathrm{mg}$ on day $143 ; \mathrm{R}: 16 \mathrm{mg}$ on day 140). In multiparous ewes, no clearcut differences in maternal care were observed between breeds, except for licking of the neonate, which started later in IF ewes than in $R$ or PA ewes $(P<0.05)$. In contrast, breed differences were much more marked in ewes lambing for the first time. Disturbances in licking or the acceptance of the lamb at the udder were less frequent in R ewes $(10 \mathrm{p} .100)$ than in IF $(50 \mathrm{p} .100)$ or PA $(35 \mathrm{p} .100)$. Aggressive behaviour was more frequent in PA females (29 p. 100 of mothers) than in IF ( 7 p. $100, P<0.05)$ or $R$ ewes $(19$ p. $100, n s) .60$ p. 100 of $R$ mothers did not show abnormal behaviour at lambing and within $3 \mathrm{~h}$ after lambing, $90 \mathrm{p}$. 100 had accepted their lamb. In IF ewes, these proportions were respectively 35 and $61 \mathrm{p} .100$ ( $\mathrm{P}<0.05$ in both cases). Performances of PA ewes were intermediate ( 34 p. 100 at lambing and 84 p. $100,3 \mathrm{~h}$ later). Thus, as a whole primiparous ewes show more frequently disturbances of maternal behaviour than multiparous mothers. Also, Romanov ewes performed better than the 2 other breeds, and Ile-de-France ewes appeared as the poorest mothers. It must be stressed also that within the 3 breeds, large individual differences were noted : some animals readily accepted their lamb whereas others consistently rejected it until the end of the observations.

Key words : Maternal behaviour, sheep, experience, breed differences.

\section{Introduction}

Chez la brebis, l'influence de la parité et de l'expérience maternelle dans la manifestation des soins aux jeunes ont été assez peu étudiées.

Quelques travaux mentionnent des différences entre brebis primipares et multipares (Alexander, 1960 ; AleXANder \& Peterson, 1961 ; Hulet et al., 1975). En particulier, ces auteurs observent que les brebis mettant bas pour la première fois ont tendance à garder l'agneau près de leur tête, ce qui peut retarder l'accès à la mamelle. Parfois, les mères primipares rejettent aussi l'agneau lors des premières tentatives de tétée. Les conséquences de ces troubles de comportement maternel sur la survie des jeunes ne sont pas clairement établies. D'après AlEXANDER (1960) et SHelley (1970), la mortalité des agneaux serait plus élevée chez les brebis primipares en partie à cause des perturbations de comportement observées à la parturition. Cependant dans une autre étude (ARNOld \& MORGAN, 1975), aucune différence marquée n'apparaît en fonction de la parité. La grande variété des résultats obtenus peut s'expliquer assez facilement, dans la mesure où ces recherches ont été effectuées sur diverses races et à l'extérieur, où de nombreux facteurs (climatiques et nutritionnels en particulier) varient d'une étude à une autre. Il est donc assez difficile, à partir de ces études, de préciser l'importance d'un facteur donné sur la manifestation du comportement maternel à la parturition.

Afin de limiter les interactions possibles avec les facteurs du milieu, nous avons donc entrepris d'étudier le comportement maternel à la parturition chez des brebis maintenues en bergerie permanente avec une alimentation contrôlée. Toutefois, ces résultats obtenus dans des conditions d'environnement précis devraient être validés dans les situations complexes qui caractérisent le milieu plus naturel de l'élevage en plein air. Nos observations ont porté sur des brebis primipares et multipares de 3 races, ce qui nous a permis d'étudier l'influence de la parité sur la manifestation du comportement maternel d'une part, et l'existence d'éventuelles différences raciales d'autre part. 


\section{Matériel et méthodes}

\section{A. Conditions générales d'entretien des animaux}

Les observations se sont déroulées à la Station de Physiologie de la Reproduction de l'I.N.R.A. de Nouzilly, ainsi qu'à la ferme expérimentale du Domaine I.N.R.A. de Brouessy. Dans ces 2 installations, les animaux sont maintenus en bergerie permanente depuis la naissance. La taille des lots était comprise entre 15 et 30 femelles (avec une densité de 0,25 à 0,5 femelle par mètre carré), les différents lots à l'intérieur d'une même bergerie étant séparés par des barrières à claire-voie. La ration des mères consistait en un apport journalier de luzerne déshydratée, avec un complément de blé, de maïs et de minéraux. De plus, les animaux disposaient de paille à volonté. La ration était ajustée afin de couvrir les besoins alimentaires des animaux en fonction du stade de gestation.

La reproduction était synchronisée aussi bien à l'ovulation qu'à la parturition. Le groupage des ovulations pour un même lot était obtenu par pose d'éponges vaginales imprégnées de progestagène, suivie d'une injection de PMSG (400 à 600 UI en fonction de la saison) au retrait de l'éponge. L'insémination était effectuée 48 heures plus tard.

\section{B. Animaux}

A l'I.N.R.A. de Nouzilly, nous avons observé des femelles de race Ile-de-France (IF), Préalpes du Sud (PA) et Romanov (R). A Brouessy, seuls des animaux de race Préalpes $d u$ Sud ont été étudiés. Les observations se sont déroulées aux mois de février, mars et avril à l'I.N.R.A. de Nouzilly, et au mois de mai à la ferme de Brouessy. Elles ont été étalées sur 2 années consécutives.

La synchronisation des parturitions a été effectuée à Nouzilly par injections de dexaméthasone (Bosc, 1974) au 144e $\mathrm{j}$ de gestation (G 144) pour les Ile-de-France (16 mg), à G 140 pour les Romanov (16 mg) et à $\mathrm{G} 143$ pour les Préalpes du Sud (12 mg). A la ferme de Brouessy, les brebis Préalpes ont été traitées avec $16 \mathrm{mg}$ de dexaméthasone à $\mathrm{G} 144$. La durée normale de la gestation est comprise entre $143 \mathrm{j}$ (Romanov) et $147 \mathrm{j}$ (Ile-de-France).

L'âge à la mise bas, chez les brebis primipares, variait de 12 à 28 mois, la majorité des mères étudiées ayant entre 13 et 18 mois au moment de la parturition (76 p. 100 des cas). Les brebis multipares ont été définies comme étant les femelles ayant mis bas au moins une fois avant l'étude. Leur âge au moment des observations était compris entre 2 et 8 ans. La moitié de ces brebis avait agnelé 2 fois ou plus avant l'étude et élevé un agneau au moins une fois. Parmi les brebis n'ayant agnelé qu'une fois (59 femelles), 31 avaient élevé leur jeune jusqu'au sevrage, entre 2 et 3 mois après la parturition. Les 28 femelles restantes avaient mis bas une seule fois et n'étaient restées en contact que 2 à $5 \mathrm{j}$ avec leur agneau. Ces dernières femelles étaient pour la plupart de race Ile-de-France (20 femelles).

Les effectifs totaux impliqués dans cette étude sont présentés dans le tableau 1. 


\section{TABleau 1}

Répartition des effectifs totaux impliqués dans l'étude du comportement maternel à la parturition, en fonction de la race et de l'expérience maternelle.

Distribution of total number involved in study of dam behaviour at parturition, as a function of breed and maternal experience.

\begin{tabular}{|c|c|c|c|c|c|}
\hline \multirow{2}{*}{ Race } & \multicolumn{2}{|c|}{ Primipares } & \multicolumn{2}{|c|}{ Multipares } & \multirow{2}{*}{ Total } \\
\hline & $\mathrm{S}$ & $\mathbf{M}$ & $\mathrm{S}$ & $\mathbf{M}$ & \\
\hline Ile-de-France (IF) & 33 & 30 & 20 & 22 & 105 \\
\hline Préalpes du Sud (PA) & $28^{\mathrm{a}}$ & $27^{\mathrm{a}}$ & $19^{b}$ & $30^{\mathrm{b}}$ & 104 \\
\hline Romanov (R) . & 3 & 22 & 5 & 29 & 59 \\
\hline Total $\ldots \ldots \ldots$ & \multicolumn{2}{|c|}{143} & \multicolumn{2}{|c|}{125} & 268 \\
\hline
\end{tabular}

$\mathrm{S}=$ portées simples $; \mathrm{M}=$ portées multiples ; (a) dont 17 femelles observées à la ferme de Brouessy ; (b) dont 16 femelles observées à la ferme de Brouessy.

$S=$ simple birth $; M=$ multiple birth; (a) including 17 females observed at Brouessy farm; (b) including 16 females observed at Brouessy farm.

\section{Méthodes d'étude - Eléments comportementaux pris en compte}

L'observateur était placé à proximité des animaux, dans le parc adjacent à celui des femelles observées. L'intervention humaine au moment de la parturition a été évitée au maximum, sauf dans les cas de mauvaise présentation de l'agneau lorsque l'assistance était indispensable. Les animaux étaient observés au minimum $30 \mathrm{mn}$ sur le lieu de mise bas avant d'être placés en cases individuelles. La grande majorité des observations se sont déroulées entre $4 \mathrm{~h}$ du matin et minuit, par suite du groupage des parturitions par la dexaméthasone.

Les observations, notées sur des fiches préparées à l'avance, peuvent être classées en 2 catégories:

1 - Cas où le comportement des animaux a été observé de façon continue pendant au minimum $30 \mathrm{mn}$ à partir de la naissance de l'agneau (ou du premier agneau dans le cas de naissances multiples).

2 - Cas où, par suite de la non disponibilité des observateurs, le comportement a été suivi de façon discontinue depuis la parturition.

Dans le premier cas, les éléments suivants ont été notés :

- temps de léchage, déterminé à l'aide d'un chronomètre, par tranches de $5 \mathrm{mn}$;

- comportement agressif de la mère ;

- comportement de la mère lorsque le jeune essaie d'atteindre la mamelle ;

- acceptation ou non de l'agneau $3 \mathrm{~h}$ après la mise bas, définie d'après l'acceptation à la mamelle et l'absence de comportement agressif de la part de la mère. Nous n'avons pas pris en compte le comportement de léchage à ce moment, puisque ce comportement évolue très rapidement, et n'est déjà plus très marqué $2 \mathrm{~h}$ après la naissance du jeune. 
Dans le second cas (animaux observés de façon discontinue) un ou plusieurs des critères suivants ont été notés : mise bas ;

- existence (et non pas durée) de léchages dans les 5 ou les $30 \mathrm{mn}$ suivant la

- comportement agressif ;

- existence ou non de troubles d'acceptation à la mamelle ;

- acceptation ou non de l'agneau $3 \mathrm{~h}$ après la mise bas.

Les 3 premiers critères retenus sont liés dans le comportement normal : léchages, absence d'agression et allaitement. Cependant, leur prise en considération indépendante résulte de l'existence de mécanismes différents pour chacun d'entre eux. Le comportement de léchage du nouveau-né résulte de la très forte attraction envers le liquide amniotique qui apparaît lors du travail (LEVY et al., 1983). Le mécanisme qui permet l'acceptation de l'allaitement est différent (PoIndron \& Le NeINDRE, 1980). Dans les 2 cas, une réaction de peur vis-à-vis du jeune peut intervenir, mais elle est indépendante de la conduite agressive.

Le choix d'un délai de $3 \mathrm{~h}$ pour l'acceptation de l'agneau permet d'apprécier la capacité de régulation des conduites de la brebis dans les conditions expérimentales. Il est cependant évident que l'épuisement rapide des réserves de l'agneau ne lui permettrait pas d'atteindre cette limite dans des conditions d'environnement défavorables.

Enfin, en limitant volontairement cette étude à la mise en place de la conduite maternelle, nous n'avons pas pris en considération le comportement différentiel vis-à-vis des jeunes issus d'une naissance multiple.

Donc, tous les éléments comportementaux n'étaient pas nécessairement répertoriés pour une même femelle. Ceci explique que le nombre d'animaux étudiés varie d'un comportement à un autre.

\section{Analyse des résultats}

Des comparaisons entre primipares et multipares ont été effectuées race par race et aussi toutes races confondues, pour chacun des éléments comportementaux pris séparément. Nous avons également effectué des comparaisons entre les différentes races. Une évolution du comportement entre 0 et $30 \mathrm{mn}$ d'une part, et 0 et $3 \mathrm{~h}$ d'autre part, a également été envisagée. Afin d'homogénéiser l'analyse des résultats entre brebis mettant bas un seul ou plusieurs agneaux, nous n'avons, dans ce dernier cas, considéré que le comportement de léchage envers le premier jeune, l'acceptation à la mamelle envers le premier agneau qui a essayé de téter, et enfin le comportement agressif vis-à-vis de n'importe lequel des agneaux. D'éventuelles différences entre mères d'une même race ayant un seul jeune et celles ayant 2 jeunes ou plus ont été recherchées par comparaison des performances dans les 2 lots ainsi définis.

Enfin, les 3 races étudiées diffèrent par certains paramètres zootechniques tels que le poids des agneaux ou la taille de la portée $(2,4 ; 1,7$ et 1,5 pour les races Romanov, Préalpes et Ile-de-France, respectivement). Nous avons donc pris en compte ces facteurs lors de la recherche de l'origine des différences notées entre les 3 races.

L'analyse statistique des résultats a été effectuée dans la majorité des cas par l'utilisation du test de $\mathrm{CHI}^{2}$ (corrigé pour 1 degré de liberté) ou par calcul des probabilités 
exactes de Fisher, d'après le programme décrit par MERSCH (1974). Les hypothèses éprouvées étant généralement de type unilatéral $\left(\mathrm{H}_{\mathrm{O}}: \mathrm{A}<\mathrm{B}\right)$ les probabilités unilatérales (notées $\mathrm{p}=$ dans le texte) sont présentées (cf. SIEGEL, 1956 et MERSCH, 1974). Lorsque d'autres tests ont été utilisés, ils sont mentionnés dans le texte.

\section{Résultats}

Dans un premier temps, nous analyserons le comportement des brebis primipares et celui des brebis multipares, toutes races confondues. Ensuite une comparaison des résultats obtenus dans les différentes races sera effectuée.

\section{A. Comportement maternel dans les 3 premières heures post-partum} chez des brebis multipares ou primipares abstraction faite du facteur race

\section{Comportement de léchage}

Seules 6 brebis sur les 122 multipares observées n'ont pas léché leur agneau dans les 5 premières mn et toutes l'on fait dans la demi-heure suivant la mise bas. Par contre, chez les brebis agnelant pour la première fois, 38 p. 100 des mères $(52 / 138)$ n'ont pas léché le nouveau-né dans les $5 \mathrm{mn}$ suivant sa naissance. Cette proportion était encore de 21 p. $10030 \mathrm{mn}$ plus tard. La différence entre brebis primipares et multipares est significative, aussi bien à $5 \mathrm{mn}$ qu'à $30 \mathrm{mn}(\mathrm{P}<0,0001)$.

Pour les brebis ayant pu être observées en continu pendant les 30 premières mn de la vie du jeune, le temps moyen de léchage par mère a été de $18,5 \mathrm{mn} \pm 0,8 \mathrm{chez}$ les multipares $(n=47)$. Chez les brebis primipares $(n=59)$, les valeurs correspondantes ont été de $14,9 \mathrm{mn} \pm 1,2$ (test $\mathrm{F}=6,4,104$ d.d.1., $\mathrm{P}<0,05$ ). Cependant, cette différence globale entre primipares et multipares est due essentiellement au fait que certaines brebis primipares n'ont pas du tout léché le nouveau-né dans les 30 premières $\mathrm{mn}$ (11/59, sỏit 19 p. 100). Si l'on exclut ces brebis, il n'existe alors plus aucune différence significative entre multipares et primipares $(18,5 \mathrm{mn} \pm 0,8$ et $18,4 \mathrm{mn} \pm 0,5)$. Il semble donc que, dans la mesure où les mères manifestent un comportement de léchage dès la parturition, les brebis primipares lèchent alors autant leur agneau que les multipares.

\section{Comportement d'acceptation à la mamelle}

Chez les brebis multipares, les agneaux ont été acceptés à la mamelle dès les premières tentatives de tétée dans la majorité des cas (110 femelles sur 115). Trois femelles seulement ont refusé leur agneau lors des tentatives de tétée pendant plus de $30 \mathrm{mn}$ (fig. 1) et toutes les brebis ont allaité leur jeune dans les $3 \mathrm{~h}$ suivant la parturition. Par contre, seulement 50 p. 100 des brebis primipares $(62 / 124)$ ont accepté l'agneau dès les premières tentatives de tétée $(P<0,0001$ vis-à-vis des multipares). Généralement, les troubles ont été caractérisés par un déplacement rapide de la mère se positionnant ensuite de façon à ce que l'agneau se trouve devant elle. Dans certains cas, le refus à la mamelle a été également accompagné de coups de tête de la part de la mère. 


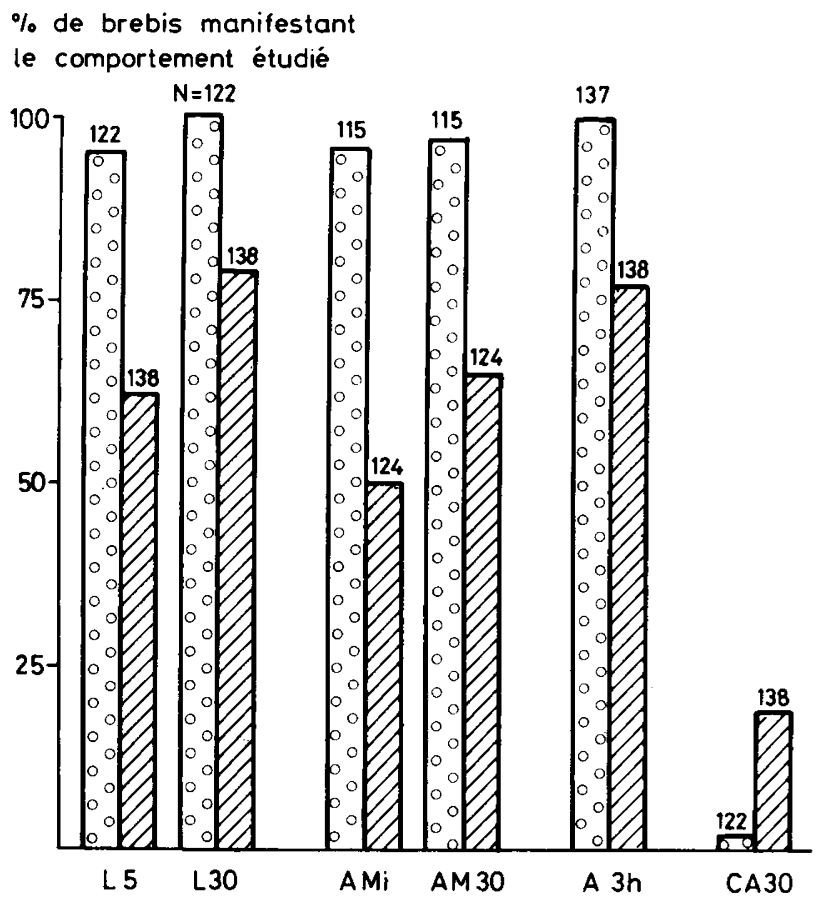

을 Brebis multipares

Z7 Brebis primipares

FIG. 1
Comparaison du comportement maternel à la parturition chez des brebis primipares ou multipares.

Comparison of maternal behaviour at parturition in primiparous or multiparous ewes.

$\mathrm{L}_{5} \quad$ : Léchage de l'agneau dans les $5 \mathrm{mn}$ suivant la naissance. Lamb licking within $5 \mathrm{mn}$ immediately following birth.

$\mathrm{L}_{30}$ : Léchage de l'agneau dans les $30 \mathrm{mn}$ suivant la naissance. Lamb licking within $30 \mathrm{mn}$ after birth.

$\mathrm{AM}_{\mathrm{i}}$ : Acceptation immédiate du jeune à la mamelle. Immediate acceptance of the newborn to the teat.

$\mathrm{AM}_{30}$ : Acceptation du jeune à la mamelle dans les $30 \mathrm{mn}$ suivant le premier essai de celui-ci. Acceptance of the newborn to the teat within $30 \mathrm{mn}$ following first attempt at sucking.

$\mathrm{A}_{3 \mathrm{~h}}$ : Acceptation du jeune $3 \mathrm{~h}$ après la naissance. Acceptance of newborn $3 h$ after birth.

$\mathrm{CA}_{30}$ : Présence de comportement agressif envers le nouveau-né dans les 30 premières mn suivant la naissance. Aggressive behaviour towards newborn within first $30 \mathrm{mn}$ after birth.

$\mathrm{N}$ : Nombre total d'animaux observés dans chaque classe ; Différence significative $(\mathrm{P}<0,05)$ entre primipares et multipares pour tous les éléments étudiés.

Total number of animals observed by category. Significant difference $(P<0.05)$ between primiparous and multiparous for all criteria studied. 
On peut noter une évolution très nette, en fonction du temps, de l'acceptation à la mamelle, chez les mères qui refusent initialement leur agneau. En effet sur 62 brebis primipares ayant manifesté un refus du jeune lors des premiers essais de tétée, 18 l'ont accepté dans les $30 \mathrm{mn}$ et 28 autres entre $30 \mathrm{mn}$ et $3 \mathrm{~h}$ (soit $77 \mathrm{p}$. 100 d'acceptation $3 \mathrm{~h}$ après la mise bas). Cette amélioration est statistiquement significative lorsqu'on compare la proportion réelle de brebis ayant changé de comportement (18/124 entre les 5 et les 30 premières minutes par exemple) à la proportion correspondant à l'hypothèse d'une absence d'évolution $(0 / 124 ; \mathrm{P}<0,0001)$.

La comparaison des naissances simples et multiples ne fait apparaître aucune différence en ce qui concerne le comportement d'acceptation à la mamelle chez les primipares $\left(\mathrm{X}_{1}{ }_{1},=0,5\right)$ et son évolution de 0 à $30 \mathrm{~mm}\left(\mathrm{X}_{1}^{2},=0,04\right)$.

\section{Comportement agressif}

Deux brebis multipares sur 122 ont manifesté un comportement agressif envers leurs agneaux dans les $30 \mathrm{mn}$ suivant la parturition. Par contre, ce comportement a été observé chez 26 des 138 brebis primipares $(\mathrm{P}<0,0001)$. Il est intéressant de souligner que d'une façon générale, le comportement agressif est apparu chez ces brebis primipares lorsque l'agneau essayait de se lever, ou bien lorsque, étant debout, il se mettait en mouvement. L'étude que nous avons réalisée n'était cependant pas assez détaillée pour que nous puissions analyser de façon précise les relations existant entre le comportement de la mère et celui du nouveau né à ce propos.

\section{Acceptation 3 heures après la parturition}

D'après les critères que nous avons définis pour l'acceptation $3 \mathrm{~h}$ après la parturition (acceptation à la mamelle et absence de comportement agressif), toutes les brebis multipares ont accepté leur agneau dans les $3 \mathrm{~h}$ (137 femelles). Par contre, chez les brebis primipares, cette proportion a été siginificativement plus faible $(106 / 138$, soit 77 p. $100 ; \mathrm{P}<0,0001)$.

\section{Absence complète de troubles de comportement maternel}

Les différents types de troubles de comportement maternel (absence de léchages, comportement agressif, rejet du jeune à la mamelle) ne sont pas toujours associés chez un même animal. Ainsi, certaines mères lèchent activement leur jeune, mais font preuve d'un comportement agressif très marqué. D'autres, bien que n'ayant commencé à lécher leur agneau que tardivement, acceptent celui-ci par la suite sans manifester d'autres troubles. Il était, par conséquent, intéressant de considérer non seulement chaque élément comportemental pris séparément, mais aussi l'ensemble de ceux-ci. Nous avons ainsi défini une classe de brebis n'ayant manifesté aucun trouble de comportement maternel. Seules, des brebis pour lesquelles nous disposions de données sur les 3 éléments comportementaux ont été prises en compte (soit 115 multipares et 124 primipares).

Chez les brebis multipares, nous retrouvons une proportion de brebis ne montrant aucun trouble qui est très proche de celle concernant l'acceptation à la mamelle $(110 / 115$ et $112 / 115$ respectivement). Par contre, chez les brebis primipares, seules 40 p. 100 des mères n'ont montré aucun trouble de comportement maternel $(50 / 124)$ dès le départ ( $\mathrm{P}<0,0001$ vis-à-vis des multipares), alors que la proportion des troubles concernant l'acceptation à la mamelle était de 50 p. 100 (paragraphe 2). Au bout de $30 \mathrm{mn}$, 60 p. 100 des mères ne manifestaient plus aucun trouble de comportement (évolution 
significative, $P<0,0001$ ) et 68 p. 100 au bout de 3 h (fig. 2). Il existe également une évolution significative entre 0 et $3 \mathrm{~h}$ chez les multipares $(P=0,04)$, mais cette évolution est beaucoup moins marquée que chez les primipares (4 p. 100 contre 28 p. 100 , $P<0,0001$, fig. 2).

$\%$ des mères observées

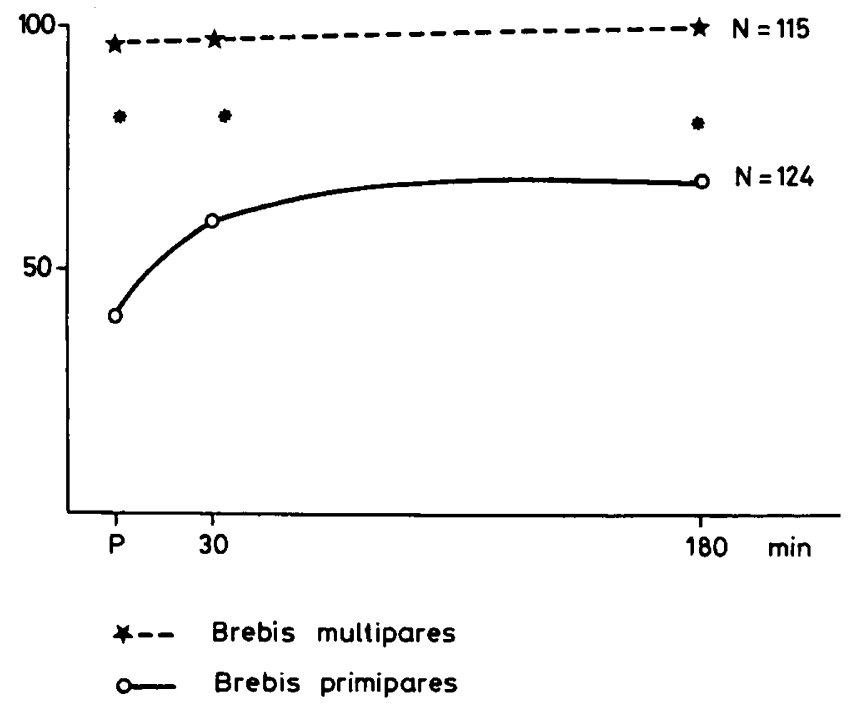

FIG. 2

Proportion de brebis primipares ou multipares ne manifestant aucun trouble de comportement maternel dans les 3 premières heures suivant la mise bas.

Proportion of primiparous or multiparous ewes that did not display any disturbed maternal behaviour within the first 3 hours following lambing.

$P$ : Dès la parturition. At parturition.

30 : Au-delà des 30 premières mn suivant la parturition. Beyond the first $30 \mathrm{mn}$ following parturition.

$180: 3 \mathrm{~h}$ après la parturition.

Différence significative $(P<0,05)$ entre les primipares et les multipares.

$3 \mathrm{~h}$ after parturition.

Significant difference $(P<0.05)$ between primiparous and multiparous.

$\mathbf{N}$ : Nombre total de mères observées dans chaque classe.

Total number of dams observed by category.

Pour résumer, il apparaît de façon très nette que les brebis primipares présentent des troubles de comportement maternel à la parturition dans plus de la moitié des cas, alors que de tels troubles sont très rares ches les multipares. Ces troubles observés chez les brebis inexpérimentées disparaissent rapidement (c'est-à-dire en moins d'1 h) chez environ la moitié des mères concernées. 


\section{B. Comparaison du comportement maternel dans les 3 heures qui suivent la parturition pour les différentes races étudiées}

\section{Brebis multipares}

Il n'existe que très peu de différences entre les 3 races prises en compte (Romanov, Ile-de-France et Préalpes $d u S u d$ ) en ce qui concerne les éléments comportementaux étudiés. Ceci s'explique aisément puisque dans la majorité des cas, aucun trouble n'est apparu chez les brebis multipares (fig. 2). La seule différence significative notée entre races concerne le début des léchages. Toutes les brebis Romanov et Préalpes ont commencé à lécher leur agneau dans les $5 \mathrm{mn}$ qui suivent la naissance ( 27 et 43 femelles respectivement) alors que 6 brebis Ile-de-France sur 42 n'ont commencé à lécher leur jeune qu'après un délai de 5 à $30 \mathrm{mn}(\mathrm{P}=0,04$ et 0,01 vis-à-vis des Romanov et des Préalpes, respectivement). Une tendance similaire a été observée en ce qui concerne l'acceptation immédiate du jeune à la mamelle. Cependant la différence n'est pas significative ( $\mathrm{P}=0,09$ et 0,12 vis-à-vis des Romanov et des Préalpes respectivement).

\section{Brebis primipares}

Lorsqu'elle est effectuée race pour race, la comparaison du comportement entre primipares et multipares fait ressortir dans l'ensemble les mêmes différences que celles relevées lors de l'analyse toutes races confondues. Les résultats par race pour les différents éléments observés chez les primipares sont représentés dans la figure 3 . Les seules exceptions notées concernent le comportement de léchage chez les brebis Romanov ainsi que le comportement agressif chez les Ile-de-France qui ne diffèrent pas en fonction de la parité. En effet, 19 primipares Romanov sur 21 ont léché leur jeune dans les $5 \mathrm{mn}$ suivant la naissance, contre $27 / 27$ chez les multipares $(P=0,18)$, et toutes l'ont léché dans les $30 \mathrm{mn}$.

Cependant, compte tenu du petit nombre d'animaux observés en race Romanov, cette absence de différence peut refléter une absence de différence comportementale en fonction de la parité, ou au contraire être due à des échantillons trop petits. Par ailleurs, dans la race Ile-de-France et à l'opposé de la tendance générale, la proportion de mères primipares manifestant un comportement agressif a été comparable à celle observée chez les multipares ( 7 p. 100 et 5 p. 100 respectivemènt, $P=0,46$ ).

A l'opposé des résultats observés chez les multipares, des différences importantes sont apparues chez les primipares en fonction de la race étudiée.

Comportement de léchage. La proportion de brebis Romanov ayant léché leur jeune dans les 5 premières mn $(19 / 21)$ est plus élevée que celle observée chez les Préalpes (33/51; $\mathrm{P}=0,02$, ou les Ile-de-France $(27 / 54 ; \mathrm{P}<0,0019)$. Par contre, la différence entre Ile-de-France et Préalpes (50 p. 100 et 65 p. 100 respectivement) n'est pas significative $(P=0,09) .30 \mathrm{mn}$ après la naissance, il n'y a plus de différence significative entre Romanov et Préalpes $(21 / 21$ et $44 / 51 ; \mathrm{P}=0,08)$, mais ces 2 races diffèrent des Ile-de-France $(36 / 54 ; \mathrm{P}=0,001$ et $\mathrm{P}=0,02$ respectivement). Ceci suggère une amélioration de comportement plus marquée chez les Préalpes que chez les Ile-de-France (fig. 3).

Acceptation à la mamelle. Bien qu'il y ait une tendance chez les Romanov à accepter plus facilement le jeune à la mamelle (13 acceptations immédiates sur 20 femelles observées) que chez les autres races, les différences ne sont pas significatives (IF : 20/44, PA : 22/48; $\mathrm{P}=0,12$ et 0,15 respectivement). Ceci est encore vrai $30 \mathrm{mn}$ après la naissance $(R=75$ p. 100 ; IF $=55$.p. 100 ; PA =65 p. 100). Comme dans le cas 
du comportement de léchage, on peut noter que la différence entre Préalpes et Ile-deFrance, initialement très faible (1 p. 100) est plus marquée à $30 \mathrm{mn}(10 \mathrm{p} .100)$ bien qu'elle ne soit pas significative $(P=0,22$; fig. 3$)$.
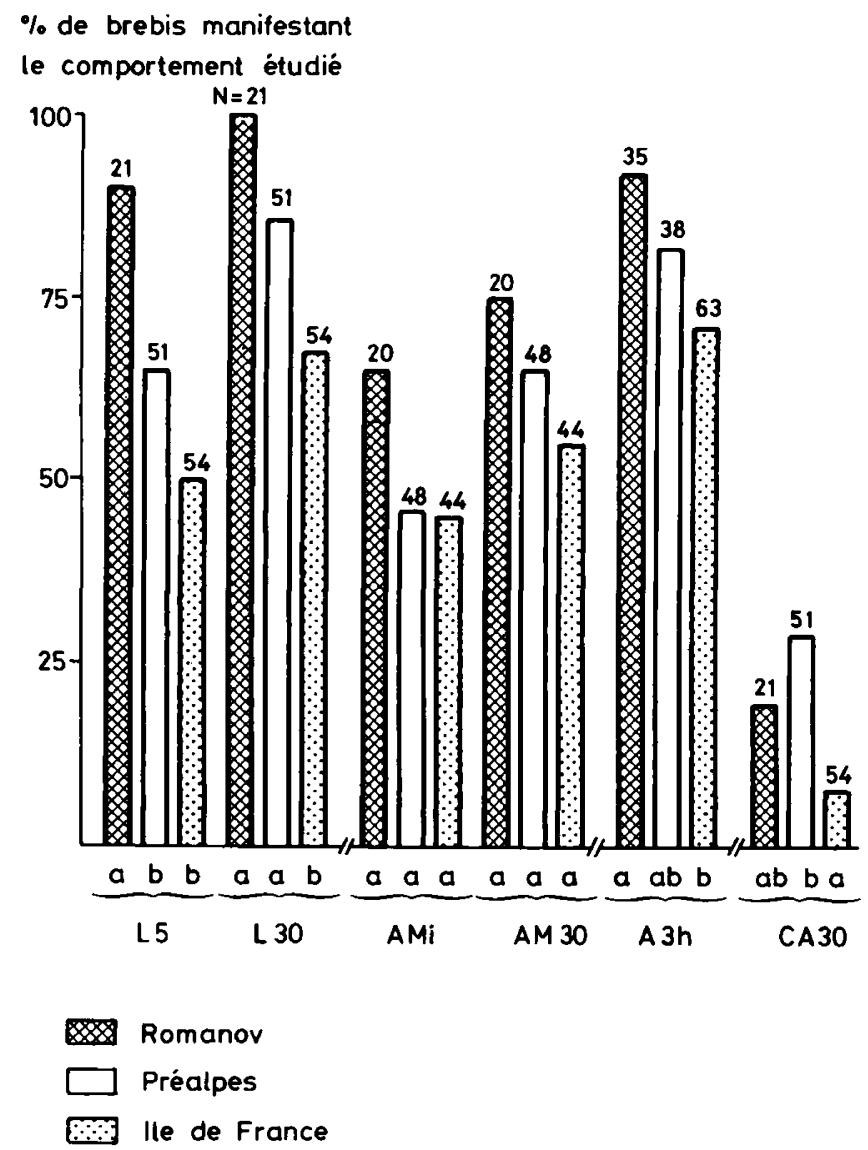

FIG. 3

Comparaison du comportement maternel à la parturition chez des brebis primipares de race Romanov, Préalpes du Sud et Ile-de-France.

Comparison of maternal behaviour at parturition in primiparous Romanov, Prealpes du Sud and Ile-de-France ewes.

$\mathrm{L}_{5}, \mathrm{~L}_{30}, \mathrm{AM}_{\mathrm{i}}, \mathrm{AM}_{30}, \mathrm{~A}_{3 \mathrm{~h}}$ et $\mathrm{CA}_{30}$ (cf. légende figure 1).

Pour chaque élément comportemental, les classes affectées d'une même lettre ne diffèrent pas significativement $(\mathrm{P}>0,05)$.

For every behavioral element, the categories affected by the same letter do not differ significantly $(P>0.05)$.

$\mathrm{N}$ : Nombre total de mères observées dans chaque classe.

Total number of dams observed by category. 
Comportement agressif. Aucune différence de comportement agressif n'a été relevée entre les primipares Romanov et Ile-de-France $(4 / 21$ vs $4 / 54 ; \mathrm{P}=0,15)$. Il en a été de même entre les Romanov et les Préalpes $(4 / 21$ vs $15 / 51 ; \mathrm{P}=0,3)$. Par contre, la proportion de brebis manifestant un comportement agressif envers le nouveau-né a été clairement plus forte chez les Préalpes que chez les Ile-de-France (15/51 vs 4/54; $P=0,005$; fig. 3).

Acceptation $3 h$ après la mise bas. La proportion de brebis acceptant leur agneau au bout de $3 \mathrm{~h}$ a été la plus élevée en race Romanov (23/25 soit 92 p. 100). Ceci ne diffère pas des résultats obtenus en race Préalpes du Sud (31/38 soit 82 p. 100 ; $P=0,21)$. Par contre, la proportion d'acceptation a été significativement plus faible en race Ile-de-France (45/63, soit 71 p. 100) qu'en race Romanov $(\mathrm{P}=0,03)$. La différence entre Ile-de-France et Préalpes du Sud n'atteint pas le seuil de signification $(\mathrm{P}=0,18)$.

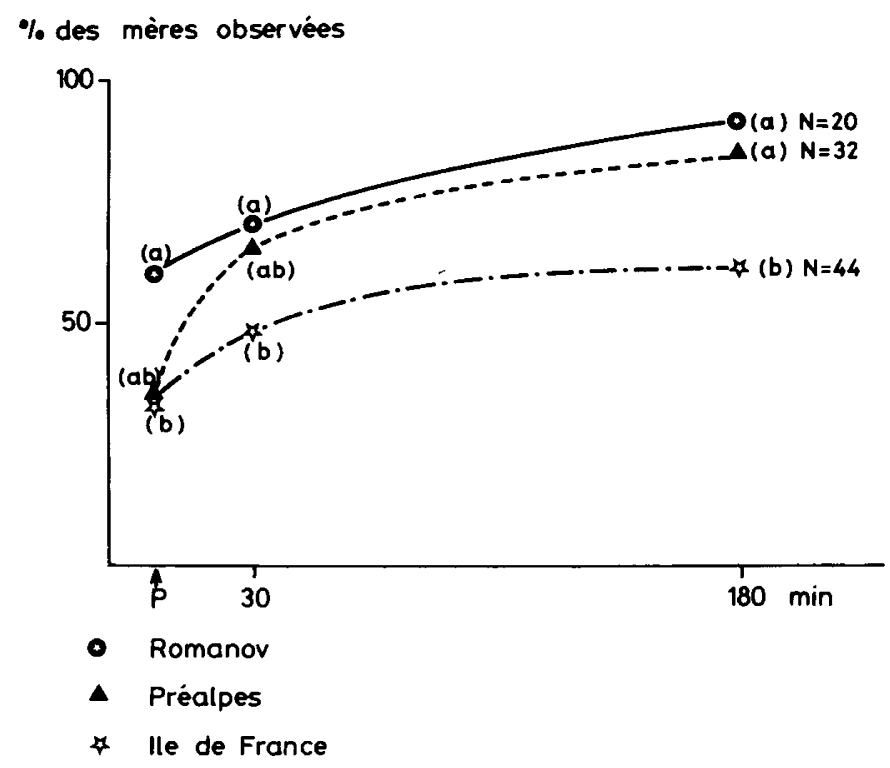

FIG. 4

Proportions de brebis primipares ne manifestant aucun trouble de comportement maternel dans les 3 premieres heures suivant la parturition en races Romanov, Préalpes du Sud et Ile-de-France.

Proportions of primiparous ewes that did not display any maternal behaviour disturbances during the first 3 hours post-partum (Romanov, Prealpes du Sud and Ile-de-France breeds).

$\mathrm{P}$ : Aucun trouble dès la parturition.

No disturbances immediately post-partum.

30 : Aucun trouble au-delà des 30 premières mn suivant la parturition. No disturbances beyond the first $30 \mathrm{mn}$ post-partum.

180 : Aucun trouble $3 \mathrm{~h}$ après la parturition.

A chaque moment $(P, 30,180)$, les classes affectées d'une même lettre ne diffèrent pas significativement $(P>0,05)$.

No disturbances $3 h$ post-partum.

At each time $(P, 30,180)$ the categories affected by the same letter did not differ significantly $(P>0.05)$. 
Absence complète de troubles de comportement maternel. Cette analyse ne concerne que les animaux pour lesquels nous disposions d'informations à la fois pour le comportement de léchage, l'acceptation à la mamelle et le comportement agressif. La combinaison des 3 facteurs comportementaux fait ressortir chez les primipares, une différence assez claire entre les Romanov d'une part et les Ile-de-France et les Préalpes d'autre part (fig. 4). 60 p. 100 des Romanov n'ont manifesté aucun trouble de comportement maternel dès la mise bas $(12 / 20)$, contre 34 p. 100 chez les Ile-de-France $(15 / 44$; $\mathrm{P}=0,05)$ et 35 p. $100 \mathrm{chez}$ les Préalpes $(11 / 32 ; \mathrm{P}=0,06)$. L'évolution la plus marquée entre 0 et $3 \mathrm{~h}$ concerne les brebis Préalpes $(+49$ p. 100), cette évolution étant significativement plus élevée que chez les Ile-de-France (+ 27 p. $100 ; \mathrm{P}=0,04)$. L'évolution observée chez les Romanov ( +30 p. 100) ne diffère pas de celle des 2 autres groupes $(\mathrm{P}=0,7$ vis-à-vis des $\mathrm{IF}$ et $\mathrm{P}=0,13$ vis-à-vis des $\mathrm{PA})$.

Les principales différences observées entre races sont rappelées dans le tableau 2.

TABLEAU 2

Principales différences entre races, de comportement maternel observées chez les brebis primipares. Main breed differences in maternal behaviour observed in primiparous ewes.

\begin{tabular}{|c|c|}
\hline Comparaisons effectuées & Nature des différences observées \\
\hline $\begin{array}{c}\text { Comparaison entre primipares } \\
\text { en fonction de la race : } \\
\text { R : Romanov } \\
\text { PA : Préalpes } \\
\text { IF : Ile-de-France }\end{array}$ & $\begin{array}{l}\text { - Proportion de brebis léchant leur agneau dans les } 5 \mathrm{mn} \text { plus } \\
\text { élevée chez les } \mathrm{R} \text { que dans les autres races }(\mathrm{P}<0,02) \\
\text { - Proportion de brebis léchant leur agneau dans les } 30 \mathrm{mn} \text { plus } \\
\text { faible chez les IF que dans les deux autres races }(\mathrm{P}<0,02) \\
\text { - Taux d'acceptation à } 3 \text { h plus élevé chez les } \mathrm{R} \text { que chez les IF } \\
\text { (P }=0,03) \\
\text { Taux intermédiaires (ns) chez les } \mathrm{PA} \\
\text { - Comportement sans aucun trouble plus fréquent chez les } \mathrm{R} \\
\text { que chez les IF ( } \mathrm{P}=0,05) \text { ou les } \mathrm{PA}(\mathrm{P}=0,06) \\
\text { - Comportement agressif plus fréquent chez les } \mathrm{PA} \text { que chez les } \\
\text { IF }(\mathrm{P}=0,05) \\
\text { Taux intermédiaires chez les } \mathrm{R}\end{array}$ \\
\hline
\end{tabular}

C. Etude des facteurs associés à la présence de troubles de comportement maternel chez les brebis primipares

Les 3 races que nous avons étudiées diffèrent par la fréquence des perturbations de comportement maternel, mais aussi par certaines caractéristiques zootechniques telles que la taille de la portée et le poids des agneaux. Ces données sont rappelées dans le tableau 3.

D'après ces données, on pourrait penser qu'il existe un lien entre la taille de la portée et le poids des agneaux, d'une part et la fréquence des troubles de comportement, d'autre part. Ceci expliquerait la différence observée dans les 3 races. Dans ce cas, une liaison similaire devrait se retrouver à l'intérieur d'une même race. Afin de la vérifier, 
nous avons entrepris de comparer dans chaque race le comportement des mères donnant naissance à un seul agneau avec celui des femelles donnant des portées multiples. Nous avons également comparé le poids des agneaux chez les mères présentant des troubles et chez celles n'en présentant pas.

\section{TABleau 3}

Principales différences zootechniques et de comportement maternel existant entre les races Romanov, Préalpes du Sud et Ile-de-France.

Performance traits and maternal behaviour of Romanov, Prealpes du Sud and Ile-de-France breeds.

\begin{tabular}{|c|c|c|c|c|}
\hline & \multicolumn{2}{|c|}{$\begin{array}{l}\text { Fréquence des troubles } \\
\text { comportementaux }\end{array}$} & \multirow{2}{*}{$\begin{array}{l}\text { Taille de la } \\
\text { portée }\end{array}$} & \multirow{2}{*}{$\begin{array}{c}\text { Poids des } \\
\text { agneaux }(\mathrm{kg})\end{array}$} \\
\hline & Parturition & à $3 \mathrm{~h}$ & & \\
\hline Romanov. & 40 p. 100 & 8 p. 100 & 2,4 & $2,2 \pm 0,1$ \\
\hline Préalpes $\ldots \ldots \ldots \ldots$ & 65 p. 100 & 18 p. 100 & 1,7 & $2,9 \pm 0,1$ \\
\hline Ile-de-France .. & 66 p. 100 & 29 p. 100 & 1,5 & $3,9 \pm 0,1$ \\
\hline
\end{tabular}

Outre ces paramètres zootechniques, le mode d'élevage des brebis dans le jeune âge peut également être impliqué, au moins si l'on se réfère aux études effectuées dans d'autres espèces telles que le singe Rhésus (HARLow et al., 1963). Aussi bien à Nouzilly qu'à Brouessy une partie des agneaux est élevée en groupe à l'allaitement artificiel, après séparation de la mère 24 à 48 h après la naissance. Ne pouvant exclure une influence éventuelle du mode d'élevage sur la maturation du comportement maternel, nous avons donc recherché des indices de son effet.

\section{Liaison avec la taille de la portée}

La comparaison des mères d'un seul agneau avec celles donnant naissance à plusieurs jeunes a été effectuée uniquement en race Préalpes et Ile-de-France, les effectifs de simples en race Romanov étant insuffisants (tabl. 1).

Au sein de la race Préalpes, aucune différence significative n'a été observée en fonction de la taille de la portée. Par contre, chez les Ile-de-France, les proportions de mères à un seul agneau ne montrant pas de perturbations des divers comportements étudiés ont été plus faibles que celles observées chez les mères de plusieurṣ agneaux (fig. 5). Ceci est vrai pour le démarrage des léchages, l'acceptation à la mamelle et l'acceptation $3 \mathrm{~h}$ après le part, mais pas pour le comportement agressif. Toutefois, les différences observées ne sont significatives que pour l'acceptation à $3 \mathrm{~h}(\mathrm{P}=0,04)$ et approchent le seuil de signification en ce qui concerne le début des léchages dans les $5 \mathrm{mn}$ suivant la naissance $(\mathrm{P}=0,08)$.

Lorsqu'on compare les 3 races entre elles, il apparaît alors que dans le cas de mères donnant naissance à plusieurs agneaux, les différences sont beaucoup plus limitées que lorsque les comparaisons entre races étaient effectuées sans tenir compte de la taille de la portée. Entre les races Romanov et Préalpes, seules les proportions de 


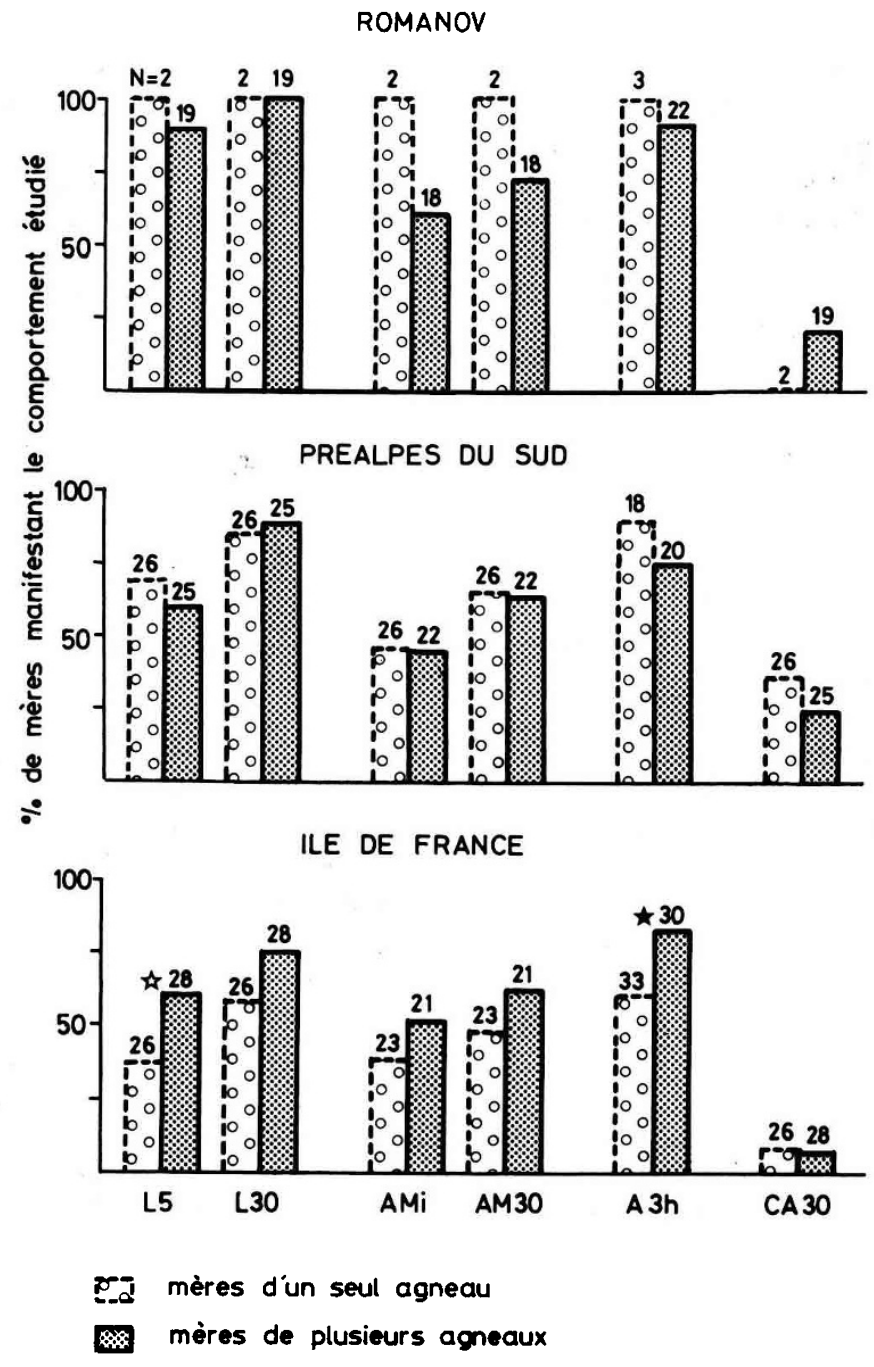

FIG. 5

Influence de la taille de la portée sur la manifestation du comportement maternel à la parturition chez des brebis primipares Romanov, Préalpes du Sud et Ile-de-France.

Influence of litter size on maternal behaviour displayed at parturition in primiparous ewes

(Romanov, Prealpes du Sud and Ile-de-France breeds).

$\mathrm{N}$ : Nombre total de brebis observées pour chaque élément comportemental.

Total number of ewes observed per behaviour pattern.

$\mathrm{L}_{5}, \mathrm{~L}_{30}, \mathrm{AM}_{\mathrm{i}}, \mathrm{AM}_{30}, \mathrm{~A}_{3 \mathrm{~h}}, \mathrm{CA}_{30}$ : cf. légende figure 1 .

*: $\mathrm{P}=0,08$

: $P=0,04$ entre mères d'un ou de plusieurs agneaux.

between dams with one or several lambs. 
brebis ayant léché leur agneau dans les $5 \mathrm{mn}$ suivant la naissance diffèrent significativement $(17 / 19$ vs $15 / 25 ; \mathbf{P}=0,03)$. Lorsqu'on compare les Préalpes et les Ile-de-France, la seule différence notable concerne le comportement agressif, plus fréquent chez les Préalpes $(6 / 25$ vs $2 / 28 ; P=0,09)$. Enfin les différences existant entre Romanov et Ile-de-France se limitent au comportement de léchage, qui apparaît plus tôt chez les Romanov (17/19 vs $17 / 28$ dans les $5 \mathrm{mn}$ et $19 / 19$ vs $21 / 28$ dans les $30 \mathrm{mn}$ suivant le part ; $\mathrm{P}=0,03$ et 0,02 respectivement). Même les taux d'acceptations $3 \mathrm{~h}$ après le part ne diffèrent pas significativement $(20 / 22$ en $R$ et $25 / 30$ en $I F, P=0,14)$ alors que les performances de ces 2 races étaient les plus éloignées dans l'analyse globale faite sans tenir compte de la taille de portée.

Par contre, lorsqu'on compare les mères d'un seul agneau, les différences raciales apparaissent de façon très marquée entre brebis Préalpes et Ile-de-France : seul le comportement d'acceptation à la mamelle ne diffère pas entre les 2 races $(P=0,41$ pour l'acceptation immédiate et $\mathrm{P}=0,17$ à $30 \mathrm{mn}$ ). Pour tous les autres éléments comportementaux, les seuils sont égaux ou inférieurs à 0,03 (fig. 5).

\section{Relation entre la présence de troubles de comportement maternel et le poids des agneaux}

Dans chaque race, nous avons comparé le poids moyen des agneaux nés de mères ayant présenté des troubles de comportement maternel avec celui des agneaux nés de mères n'ayant manifesté aucune perturbation. Les résultats de cette analyse sont résumés dans le tableau 4. Seule une différence est apparue en ce qui concerne le poids des agneaux chez les brebis Ile-de-France ayant ou non accepté leur agneau $3 \mathrm{~h}$ après la parturition, les agneaux des mères ayant présenté des troubles étant plus lourds que ceux des mères n'ayant eu aucun trouble $(4,24 \pm 0,2 \mathrm{~kg}$ vs $3,56 \pm 0,12 \mathrm{~kg}, \mathrm{P}<0,01)$.

\section{TABLEAU 4}

Comparaison du poids des agneaux chez des brebis primipares

en fonction de la présence ou non de troubles de comportement maternel à la parturition.

Comparison of lamb weight in primiparous ewes according to maternal behaviour at parturition.

\begin{tabular}{|c|c|c|c|c|c|}
\hline \multirow{2}{*}{\multicolumn{3}{|c|}{ Race }} & \multicolumn{2}{|c|}{ Poids des agneaux chez les brebis } & \multirow[b]{2}{*}{ Test de $\mathbf{F}$} \\
\hline & & & $\begin{array}{l}\text { n'ayant manifesté } \\
\text { aucun trouble } \\
\text { de comportement }\end{array}$ & $\begin{array}{l}\text { ayant manifesté } \\
\text { des troubles } \\
\text { de comportement }\end{array}$ & \\
\hline Romanov & $\cdot$ & $\begin{array}{l}\text { A } \\
\text { B }\end{array}$ & $\begin{array}{l}1,99 \pm 0,10 \\
2,00 \pm 0,08\end{array}$ & $\begin{array}{l}2,12 \pm 0,10 \\
2,38 \pm 0,10\end{array}$ & \multirow{3}{*}{$\begin{array}{c}\text { ns } \\
\text { ns } \\
\text { ns } \\
\text { ns } \\
\text { ns } \\
\mathrm{P}<0,01\end{array}$} \\
\hline Préalpes & & $\begin{array}{l}\text { A } \\
\text { B }\end{array}$ & $\begin{array}{l}2,71 \pm 0,15 \\
2,73 \pm 0,11\end{array}$ & $\begin{array}{l}2,71 \pm 0,14 \\
2,61 \pm 0,35\end{array}$ & \\
\hline Ile-de-France & . & $\begin{array}{l}\text { A } \\
\text { B }\end{array}$ & $\begin{array}{l}3,82 \pm 0,19 \\
3,56 \pm 0,12\end{array}$ & $\begin{array}{l}3,73 \pm 0,14 \\
4,24 \pm 0,20\end{array}$ & \\
\hline
\end{tabular}

A : Présence ou absence de troubles comportementaux à la parturition.

B : Présence ou absence de troubles comportementaux $3 \mathrm{~h}$ après la parturition. 
Ceci recoupe l'analyse concernant la taille de la portée, puisque d'une façon générale, les agneaux simples sont plus lourds que les jumeaux ou les triplets. Par contre, ce résultat ne se retrouve pas dans les autres races.

Par ailleurs, il convient de rappeler que dans toutes les races, et même chez les Ile-de-France, des troubles ont été notés dans une proportion non négligeable de mères de jumeaux ou de triplets (12 mères par exemple en race IF).

\section{Relation entre le mode d'élevage des mères dans le jeune âge}

et la manifestation de troubles de comportement maternel

Nous avons comparé les taux d'apparition des troubles comportementaux chez les brebis élevées avec leur mère et chez celles élevées en allaitement artificiel. De plus, nous avons aussi vérifié si, chez les brebis manifestant des troubles, la proportion de brebis élevées selon un mode d'élevage donné différait de celle existant chez les brebis ne présentant aucun trouble de comportement maternel. Les données sont rassemblées dans le tableau 5. D'après ces analyses, il ne semble y avoir aucune relation entre le mode d'élevage au cours de la phase d'allaitement et l'existence de troubles de comportement maternel à la parturition.

\section{TABleau 5}

Répartition des troubles de comportement maternel à la parturition chez les brebis primipares en fonction du mode d'élevage dans le jeune âge.

Distribution of disturbed maternal behaviour at parturition in primiparous ewes as a function of rearing system in early life.

\begin{tabular}{|c|c|c|c|c|}
\hline \multirow{2}{*}{ Race } & \multirow{2}{*}{ Nature des troubles } & \multicolumn{2}{|c|}{ Allaitement } & \multirow{2}{*}{$\begin{array}{c}\text { Test de } \\
\text { comparaison }\end{array}$} \\
\hline & & maternel & artificiel & \\
\hline Romanov . & $\begin{array}{l}\text { - à la parturition } \\
\text { - à } 3 \mathrm{~h}\end{array}$ & $\begin{array}{l}5 / 13 \\
1 / 16\end{array}$ & $\begin{array}{l}2 / 8 \\
1 / 9\end{array}$ & $\begin{array}{l}0,44 \\
0,60\end{array}$ \\
\hline Préalpes . & $\begin{array}{l}\text { - à la parturition } \\
\text { - à } 3 \mathrm{~h}\end{array}$ & $\begin{array}{r}11 / 21 \\
6 / 22\end{array}$ & $\begin{array}{r}10 / 14 \\
2 / 16\end{array}$ & $\begin{array}{l}0,22 \\
0,25\end{array}$ \\
\hline Ile-de-France & $\begin{array}{l}\text { - à la parturition } \\
\text { - à } 3 \mathrm{~h}\end{array}$ & $\begin{array}{l}21 / 33 \\
11 / 41\end{array}$ & $\begin{array}{r}12 / 18 \\
9 / 20\end{array}$ & $\begin{array}{l}0,54 \\
0,13\end{array}$ \\
\hline Total & $\begin{array}{l}\text { - à la parturition } \\
\text { - à } 3 \mathrm{~h}\end{array}$ & $\begin{array}{l}36 / 67 \\
18 / 79\end{array}$ & $\begin{array}{l}24 / 40 \\
12 / 45\end{array}$ & $\begin{array}{l}0,39 \\
0,39\end{array}$ \\
\hline
\end{tabular}

(a) Probabilité (exacte de FISCHER) que les proportions observées ne diffèrent que du seul fait du hasard.

\section{Discussion}

La comparaison du comportement maternel chez des brebis primipares et multipares permet de souligner les différences existant en fonction de la parité des mères. Alors que chez les brebis multipares, les perturbations comportementales sont rares, la moitié environ des mères primipares manifestent des troubles plus ou moins marqués allant 
d'un retard dans le début des léchages jusqu'à une absence totale d'intérêt pour le nouveau-né. Nos résultats sont en accord avec ceux d'AlEXANDER (1960) et de Hulet et al. (1975), qui rapportent des différences assez marquées entre brebis primipares et multipares en race Mérinos. De plus, compte tenu des conditions dans lesquelles ont eu lieu nos observations, on peut exclure une influence des facteurs climatiques ou nutritionnels. Les différences liées à la parité que nous rapportons ici sont par contre beaucoup plus marquées que celles citées par ARNOLD \& MORGAN (1975). Toutefois ces auteurs, s'ils précisent l'âge des animaux observés ( 2 ans pour les femelles les plus jeunes), ne donnent pas d'indications sur la parité exacte des mères. Il en est de même dans l'étude de SHELley (1970), qui rapporte des différences assez minimes entre jeunes et vieilles brebis. Or, une telle précision est importante puisqu'il semble exister une très nette amélioration du comportement maternel entre la $1^{\text {re }}$ et la $2^{\mathrm{e}}$ parturition, au moins dans nos conditions d'élevage. En effet, la moitié des brebis multipares que nous avons étudiées n'avaient eu l'expérience que d'une seule parturition avant l'étude, et pourtant la fréquence des troubles temporaires n'a été que de 6 p. 100 chez ces mères. De plus aucune femelle de ce type n'a rejeté son jeune de façon permanente.

L'étude des différences de comportement maternel entre les 3 races dont nous disposions est également intéressante à plusieurs points de vue. Elle souligne d'abord que ces différences ne sont pas observables sur tous les types d'animaux. On ne les trouve pratiquement pas chez des animaux multipares, alors qu'elles sont très marquées chez des brebis primipares. Par conséquent, l'expérience et surtout le nombre de mise bas sont des éléments qui doivent être pris en compte dans l'étude des différences raciales de comportement maternel chez les ovins. Ceci explique peut-être en partie les faibles différences raciales observées par ARNOLD \& MORGAN (1975). L'influence de la parité est d'ailleurs importante aussi dans l'étude des différences individuelles : alors que des différences extrêmes existent entre primipares d'une même race, ces différences sont minimes, voire inobservables chez des brebis ayant déjà mis bas avant l'étude. Ces points sont donc importants à considérer si l'on veut effectuer une sélection du comportement maternel chez les ovins.

D'après nos résultats sur les primipares, les brebis Romanov seraient celles qui, globalement, présentent le moins de perturbations de comportement. D'autre part, si les proportions de brebis Ile-de-France et Préalpes manifestant des troubles sont similaires juste après la parturition, l'amélioration ultérieure est beaucoup plus marquée chez les Préalpes que chez les Ile-de-France. Ce sont donc en définitive ces dernières qui montrent le plus de difficultés à accepter leur agneau dans les $3 \mathrm{~h}$ suivant le part. Ceci est vrai bien que les brebis Préalpes soient plus agressives envers leur jeune que les Ile-de-France. Il semblerait d'une façon schématique que chez les femelles Préalpes, les troubles se traduisent principalement par un comportement ambivalent d'attrait pour le nouveau-né associé à un refus temporaire. Par contre, chez les Ile-de-France, il s'agirait plus souvent d'un manque complet d'intérêt envers le jeune. Les quelques cas de perturbations observés en race Romanov suggèrent que ces femelles sont assez proches des brebis Préalpes.

Les causes exactes des troubles comportementaux observés ne sont pas connues. De nombreux facteurs peuvent être impliqués. Il est probable que l'expérience maternelle joue un rôle important dans l'amélioration du comportement maternel d'une parturition à la suivante. Plusieurs arguments sont en faveur d'une telle hypothèse. D'une 'part, l'amélioration du comportement maternel de nombreuses mères primipares dans les heures qui suivent la parturition illustre le fait que la mère " apprend " à s'occuper de sa progéniture. D'autre part, on sait que le déclenchement du comportement maternel, que ce soit à l'aide d'un traitement hormonal (LE NEINDRE et al., 1979) ou bien chez 
la brebis gestante à quelques jours du terme (PoINDron \& LE NEINDRE, 1980) est beaucoup plus aisé chez des brebis expérimentées que chez des brebis naïves. Ces résultats indiquent que l'expérience maternelle facilite la manifestation du comportement maternel et pourraient expliquer les difficultés plus fréquentes rencontrées chez les primipares.

Toutefois, l'expérience maternelle n'est sans doute pas le seul facteur responsable des perturbations comportementales observées chez les primipares. Ce facteur ne permet de rendre compte ni des variations observées entre races, ni des différences individuelles observées au sein d'une même race. Des paramètres comportementaux non directement liés au comportement maternel ainsi que des facteurs non comportementaux peuvent également être invoqués. Par exemple, une différence d'émotivité en fonction de l'âge pourrait, si elle existe, expliquer les réactions différentes des brebis primipares et multipares, dans la situation nouvelle qu'est la présence soudaine d'un nouveau-né après les difficultés de la mise bas.

Des différences d'émotivité pourraient de la même façon intervenir dans la manifestation de différences individuelles ou raciales. Des paramètres physiologiques peuvent également influencer la manifestation du comportement maternel. La dexaméthasone, en accélérant le processus de la mise bas peut conduire à une parturition plus pénible chez des femelles agnelant pour la première fois que chez des brebis multipares.

Toutefois, les mécanismes de mise en place du comportement maternel paraissent identiques lorsque la parturition est induite par la dexaméthasone (POINDRON \& LE NEINDRE, 1980). On sait qu'une parturition longue ou dystocique a des effets néfastes sur l'acceptation du jeune (Wallace, 1949 ; ARNOLD \& MORGan, 1975). Il est également possible que des différences non connues dans la physiologie hormonale existent entre primipares et multipares, ou entre primipares des 3 races étudiées. Dans la mesure où la motivation maternelle est en partie sous dépendance hormonale, de telles différences pourraient influencer la manifestation des soins aux jeunes à la parturition.

Enfin parmi les autres facteurs susceptibles d'influencer le comportement maternel, le poids de l'agneau et/ou la taille de la portée apparaissent comme des éléments importants, au moins en race Ile-de-France. La fréquence élevée des troubles dans les portées de simples de cette race (ou bien chez les mères d'agneaux lourds) suggère que les différences observées entre les brebis Ile-de-France, d'une part, et les Préalpes ou les Romanov, d'autre part, sont dues en grande partie à des difficultés de mise bas, même si celles-ci ne sont pas suffisamment marquées pour parler de dystocie.

Il serait nécessaire, dans les études ultérieures, de relier les variations du déroulement du processus de la parturition aux diverses formes de perturbations que nous avons décrites en ce qui concerne la mise en place du comportement maternel.

Pour conclure, il apparaît que de nombreux facteurs sont susceptibles d'intervenir, probablement en interaction, pour influencer la manifestation du comportement maternel. L'étude des brebis primipares paraît particulièrement intéressante puisque c'est chez les femelles inexpérimentées que les effets des différents paramètres sont le plus facilement observables. Cependant, une parfaite connaissance des mécanismes de contrôle de la motivation maternelle chez la brebis primipare est nécessaire pour mieux comprendre comment les différents facteurs agissent et pour pouvoir préciser la signification des différences raciales et individuelles décrites ici.

Reçu le 27 octobre 1983. 


\section{Références bibliographiques}

Alexander G., 1960. Maternal behaviour in the Merino ewes. Proc. Aust. Soc. Anim. Prod., 3, 105-114.

AleXander G., Peterson J.E., 1961. Intensive observations during lambing in a flock of maiden Merino ewes. Aust. Vet. J., 37, 371-381.

Arnold G.W., Morgan P.D., 1975. Behaviour of the ewe and lamb at lambing and its relationship to lamb mortality. Appl. Anim. Ethol., 2, 25-46.

BosC M.J., 1974. La parturition provoquée chez les mammifères domestiques. In : Bosc M.J., Palmer R., Surreau C. (éd.), Avortement et parturition provoqués, 1-24, Masson, Paris.

Harlow H.F., Harlow M.K., Hansen E.W., 1963. The maternal affectional system of Rhesus Monkeys. In : Rheingold H.L. (ed), Maternal behavior in mammals, 254-281, J. Wiley and sons, New York.

Hulet C.V., Alexander G., Hafez E.S.E., 1975. The behaviour of Sheep. In : Hafez E.S.E. (ed.), The Behaviour of domestic animals, 246-294, Baillière Tindall, London.

Le Neindre P., Poindron P., Delouis C., 1979. Hormonal induction of maternal behavior in non-pregnant ewes. Physiol. Behav., 22, 731-734.

LeVy F., Poindron P., Le Neindre P., 1983. Attraction and repulsion by amniotic fluids and their olfactory control in the ewe around parturition. Physiol. Behav., 31, 687-692.

Mersch G., 1974. Tables de contingences $2 \times 2$ pour les petits échantillons. Rev. Stat. Appl., 22, 69-81.

Poindron P., Le Neindre P., 1980. Endocrine and sensory regulation of maternal behavior in the ewe. Adv. Stud. Behav., 11, 75-119.

SHELLEY L., 1970. Interrelationships between the duration of parturition, post-natal behaviour of ewes and lambs and the incidence of neonatal mortality. Proc. Aust. Soc. Anim. Prod., 8, 348-352.

SIEGEL S., 1956. Non parametric statistics for the behavioral sciences. Mc Graw-Hill, New York, $312 \mathrm{p}$.

Wallace L.R., 1949. Observation of lambing behaviour in ewes. Proc. N.Z. Soc. Anim. Prod., 9, 85-96. 